\title{
El orden \\ de la memoria
}

Rodrigo Witker

La museología mexicana, aquella que se preocupa diariamente por la preservación y difusión de tal o cual colección, que advierte de los riesgos en que se encuentran diversos patrimonios de nuestra sociedad, que estudia y propone diversas maneras de apropiarse e interpretar a los bienes culturales y naturales, al parecer se ha vuelto insensible a uno de los acervos más preciados que debiera custodiar. Me refiero a los bienes culturales que constituyen y componen su propia memoria, su propio patrimonio, aquellos documentos que testimonian y evidencian las maneras de hacer y construir museografia en nuestro país. Esta paradoja es evidente. El simple hecho de asignar más importancia a las funciones de rescatar, ordenar, interpretar y representar públicamente los temas y los bienes de la cultura material que constituyen la memoria común de diversas colectividades, que a las de concentrar, ordenar e interpretar lo correspondiente a su propia memoria, la memoria de la comunidad museográfica, asi lo refleja.

Cuando hablamos de memoria museográfica, al menos para este trabajo y conscientes de que el espectro que abarca es mucho más amplio, nos referimos a la concentración, tanto de fuentes y documentos originales que integran a los proyectos museográficos como a las herramientas, los procesos y, principalmente, las experiencias que han sido desarrolladas en México al momento de construir los museos. Y la idea es entender que no se trata únicamente de concentrar documentos, como ocurre en algunos centros de documentación, sino que, aparte de dicho acopio, y por encima de todo, la intención es darle sentido a dicha reunión de materiales, proponerle órdenes a estas agrupaciones con la intención de que los datos obtenidos no solamente sean coleccionados y preservados, sino que, por el contrario, nos brinden información con la cual poder evaluar y poder construir a partir de corregir lo realizado.

Uno de nuestros supuestos de acción significa comprender que cada una de estas fuentes o documentos conlleva información valiosa que nos remite a las experiencias de trabajo llevadas a cabo en cada proyecto museográfico. Estas experiencias pueden ser muy variadas y podrán referirse ya sea a procedimientos, prácticas o politicas, las cuales, generalmente, desechamos sin reparar en ellas. 
Para este trabajo consideramos a la "experiencia museográfica" (relaciones que se dan entre objeto y sujeto en un sistema determinado) como una forma muy específica de conocimiento, que bien puede ser observada para sacar provecho de ella porque la consideramos relevante, ya sea por sus aportaciones como por sus limitantes y, porque, quizá lo más importante, cuando la sometemos a procesos de sistematización y clasificación, se convierte en una eficaz herramienta que permite analizar, desde perspectivas transdisciplinares, una serie de fenómenos que le son propios, gracias a los cuales podemos dar respuestas comprobadas a los problemas que se presenten de planeación museográfica. Este principio nace de considerar que a medida que va avanzando el conocimiento, el sujeto que conoce impregna al objeto de sus determinaciones, por lo que, al transcurrir el tiempo, más del sujeto está en el objeto, a la vez que, más del objeto está en el sujeto. Esto significa que el proceso del conocimiento nos permite ir construyendo verdades cada vez más complejas'.

Al observar con detenimiento estas experiencias, o conjunto de experiencias, nos damos cuenta de que siempre corresponden a escenarios o circunstancias muy particulares, los que también forman parte del sistema previamente propuesto, para los cuales, al momento de que se le propone una estructura teórica que los sustente, se convierten en conocimientos que bien pueden transformarse en métodos, técnicas o herramientas y que corresponderán a momentos decisivos de un proceso de planeación museográfica.

Nuestra intención es aprender de todo ello, es decir, concentrar información y ordenarla de forma tal que, resultado de su sistematización y análisis, podamos aprender de ella. La premisa es que podemos aprender de las experiencias, museográficas en este caso, para proponerlas como puntos de referencia e información que nos permitan, entre otros, aportar tanto en los procesos de creación de nuevos museos y exposiciones como en los de formación y consolidación de profesionales en la materia.

Las explicaciones son entonces, y tomando a Humberto Maturana, ${ }^{2}$ proposiciones que intentan reformular o recrear las observaciones de un fenómeno en un sistema de conceptos aceptables para un grupo de personas que comparten un mismo criterio de validación. En ellas se describen los fenómenos y se propone un sistema conceptual que sea capaz de generar el fenómeno a explicar de una manera aceptable para la comunidad de observadores. Este sistema conceptual debe permitir deducir, además, otros fenómenos no considerados explícitamente en la proposición original, así como describir las condiciones de observación en la comunidad de observadores. Las explicaciones siempre deben tener una referencia empírica, o sea, deben venir de la realidad; deben ser intersubjetivas, o sea, rebasar el nivel fenomenológico; deben ser sistemáticas, o sea, demostrar cómo fueron construidas y cómo se llegaron a esas conclusiones y, deben, también, ser falsables, o sea, cuestionables.

1 Pensamiento complejo según los planteamientos de Edgar Morin y de quienes comparten esas maneras de definir, intepretar y explicar la realidad, me refiero a Humberto Maturana, Francisco Varela y Niklas Luhmann, entre otros.

2 Apuntes de los cursos: Construcción de objetos de estudios y Alquimia tecnológica impartidos por el Dr. Jorge González como parte de las actividades del Laboratorio de Comunicación Compleja, México, UIA, 2000. 


\section{Programa Memoria Museológica Mexicana (MMM)}

Para esto creamos un programa de cómputo Memoria Museológica Mexicana (MMM) que, por medio de diversas formas y niveles de consulta, permite concentrar y, paralelamente, identificar, en las mismas fuentes originales, cómo han sido construidos (estrategias y recursos) los museos y sus exposiciones en México, con particular énfasis en los últimos veinte años.

La intención ha sido, tratar de conocer y explicarnos, al mismo tiempo, cómo se construyen explicaciones a partir de observar, sistematizar y reflexionar sobre un conjunto de experiencias museográficas que se han llevado a cabo recientemente en seis diferentes proyectos de nuestros pais con el deseo de hacer más eficientes los procesos de planeación museográfica.

\section{La muestra}

De acuerdo con esto, hicimos el acopio, la clasificación y la sistematización de materiales (fuentes y documentos originales) correspondientes a seis museos realizados durante la última década en nuestro país. La intención es que ellos nos sirvan, en una primera instancia. como base para proponer una estructura básica de operación (mapa de navegación) del programa, la que se verá enriquecida, posterior y permanentemente, con la inclusión de otros ejemplos más.

Museo de Palenque, Chiapas, 1993: La importancia de este proyecto radica en el carácter ex profeso de su edificio de tipo arqueológico, de financiamiento público y de museo de sitio, ya que se encuentra ubicado en la misma zona arqueológica. Ha sido reestructurado museográficamente en tres ocasiones: en 1992, 1993 y 2002.

Museo del Pueblo Maya, Dzibilchaltún. Mérida, Yucatán, 1994: La estructura de este museo arqueológico fue realizada por el arquitecto González Gortazar. Es un edificio ex profeso que es administrado por el INAH y se encuentra en la misma zona arqueológica. El proyecto museográfico estuvo a cargo del profesor Miguel Ángel Fernández.

Museo de Historia Mexicana, Monterrey, Nuevo León, 1994: Ubicado también en un inmueble ex profeso, forma parte de la oferta turística de la céntrica zona de la macroplaza en la capital del estado. El proyecto museográfico fue dirigido por el arquitecto Jorge Agostoni y el proyecto correspondió a los arquitectos Álvarez y Bulnes.

Museo del Desierto, Saltillo, Coahuila, 1999: Proyecto realizado desde 1996 hasta fines de 1999 en que es inaugurado. El edificio, ex profeso, fue realizado por el arquitecto López Guerra y responde a un intenso y exhaustivo trabajo interdisciplinario de sus contenidos museográficos. Es un museo de historia natural 
con énfasis en la paleontología donde la interactividad juega un papel importante:

Museo Nacional de Arte, México, DF. 2000: La documentación disponible corresponde al Plan Maestro, a la campaña de obtención de fondos y al proyecto de rescate y reacondicionamiento realizados durante los años 1999 y 2000. Se trata de un edificio histórico que fue reestructurado para exponer sus colecciones de arte. Es un museo nacional y de financiamiento público.

Museo Arocena, Torreón, Coahuila, 2003: Adaptación arquitectónica y museográfica de un inmueble histórico. Exposiciones de arte y de historia regional abiertas al público a mediados de 2005 , de carácter privado. La documentación disponible corresponde a las fases de planeación museológica y museográfica, guiones, zonificación temática y arquitectónica, presupuestos y procesos de producción.

\section{¿Qué puede explicar el Programa MMM?}

La revisión y sistematización de los documentos de estos seis museos nos permiten proponer cuatro iniciales maneras de dar respuesta a la interrogante central del proyecto. La primera hace referencia a que, gracias a la reflexión, a la fundamentación y argumentación empleada al momento de construir nuevos museos en México, se han ido construyendo -quizá de manera muy inconsciente- una serie de conocimientos propios de la teoría museológica que, basados en particulares condiciones, nos hablan de políticas y procedimientos empleados en cada etapa de planeación y de la evaluación de las mismas. La segunda, muy ligada a la anterior, que tiene que ver con la definición y el uso que se hace, a nivel profesional, de diversos términos, digamos que un lenguaje técnico, con los cuales se ha ido creando la museografia nacional. La tercera que nos permite conocer y evaluar el uso y el manejo que se ha hecho de los diversos recursos y herramientas museológicas y de las circunstancias en que han sido utilizadas, y la cuarta, que ofrece información correspondiente a la opinión, tanto pública como especializada, que se ha generado en torno a la edificación, construcción y puesta en funcionamiento de nuevos museos en nuestro país.

\section{La primera: Reflexión y teoría museológica}

Esta surge de la concentración de todos los documentos que dan sustento, justifican y fundamentan la creación de los nuevos museos. En ellos encontramos argumentos que nos dicen, por ejemplo ¿cómo nos hemos apropiado de los diversos conceptos y definiciones de museo?, ¿quién y cómo se los define y clasifica?, ¿cómo entiende y estudia, cada museo, a sus públicos reales o potenciales?, ¿cuáles son las formas de interpretación y representación que se han utilizados para comunicarse con sus públicos?, etcétera.

Para este caso, el programa MMM permite la consulta de la información por medio de un menú llamado búsqueda por proyecto. En este nivel, todos los documentos disponibles son convertidos en archivos digitales y clasificados de manera jerárquica y lineal. Se respeta el 

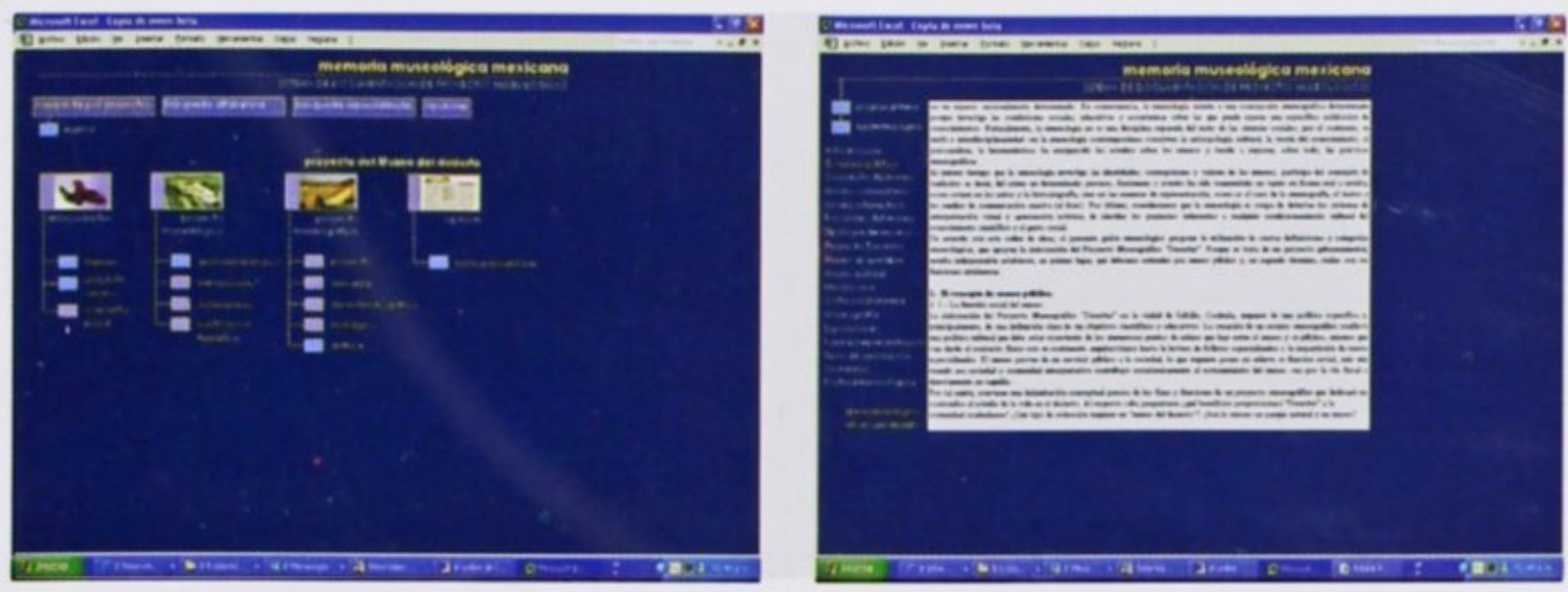

orden cronológico y secuencial que tuvo cada proyecto, aspecto que nos permite evidenciar, por ejemplo, similitudes y pautas que pudieron haber sido comunes entre todos a lo largo del tiempo.

En esta primera categoria el programa $\mathrm{MMM}$ permite, fundamentalmente, conocer de políticas y procedimientos que han sido utilizados en México, según lo ha requerido cada situación particular (origen público o privado de los recursos, del inmueble y las colecciones). Si entendemos por política aquella que define y que comprende a los compromisos futuros (funciones museísticas) que una institución debe desarrollar a partir de su misión, mandato y propósitos para cumplir con sus objetivos previamente establecidos, podemos decir, por lo tanto, que existen muchos tipos de políticas: para las colecciones, para la conservación de las colecciones y el inmueble, para las formas de interpretación y representación, para las relaciones públicas, etcétera. Los procedimientos, por su parte, son la forma sistemática de llevar a cabo cada una de dichas funciones museísticas. La clasificación de la información en esta categoría nos permite, entonces, generar o enriquecer las tipologías museológicas que cotidianamente manejamos para definir a los museos o, más específico aún, sugerir esquemas clasificatorios para los diferentes procesos, políticas y procedimientos que desarrollan al momento de crear museos.

Para comprobar esto, el Programa MMM ofrece, entre otros y como ejemplo de política para la creación inmuebles, la documentación del proceso que se siguió para la construcción del Museo del Desierto, Saltillo, 1999, en el cual, posterior a un concurso arquitectónico abierto y a nivel nacional, que sentaba las bases de su diseño a partir de necesidades museológicas, el arquitecto López Guerra (ganador del concurso) construyó los 4,200 $\mathrm{m}^{2}$ en los que actualmente se asienta el museo. Ahora, como ejemplo de política de readaptación de inmuebles, el programa MMM ofrece tanto la documentación del Proyecto Munal 2000 como la del Proyecto Museo Arocena, ambos ubicados en edificios históricos cuyos proyectos debieron modificar las funciones arquitectónicas originales para transformarlas a nuevos usos. Los dos proyectos revisten importancia debido a que, por ejemplo, en cuanto al origen de los recursos (Marco Institucional), el primero de fondos públicos y el segundo de origen privado, existen marcadas diferencias que condicionan al momento de diseñar sus contenidos. 

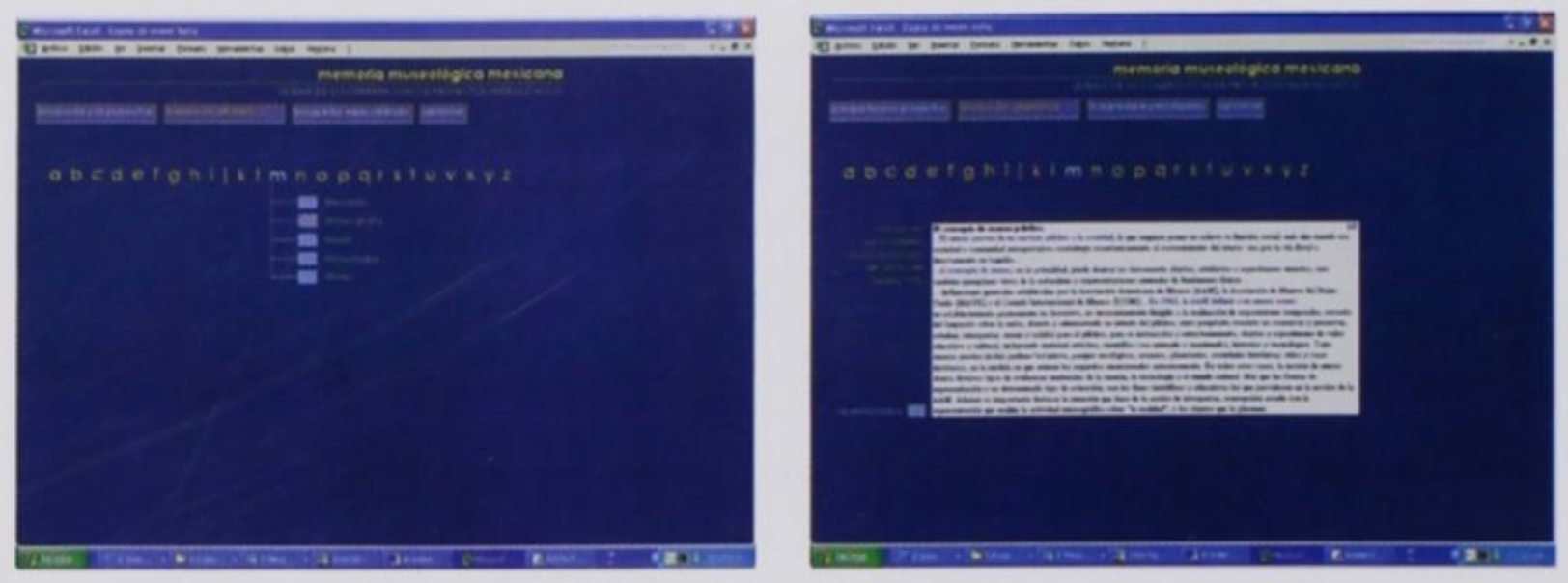

La segunda: Lenguaje museográfico

Todos los documentos concentrados en esta categoría nos dan cuenta de cómo se han ido construyendo las definiciones y cómo han sido utilizados los términos para clasificar el conocimiento museológico en los últimos años, principalmente el referido a los aspectos prácticos y operativos del trabajo museográfico. Estos elementos, sumados a los anteriores. forman parte sustancial de este llamado lenguaje museográfico.

Para acceder, el programa MMM ofrece la opción de búsqueda de manera alfabética. Ésta nos permite consultar la información por medio de palabras o conceptos. Se convierte en una especie de lexicón o diccionario de términos museológicos. La riqueza consiste en que las fuentes de donde se pueden obtener dichas definiciones y términos son amplias ya que pueden estar contenidas tanto en los guiones como en los cedularios o en documentos similares de cada proyecto. En ellos podemos ver, por ejemplo, cómo han sido utilizados elementos claves como: la extensión de los textos, la cantidad de palabras y líneas de textos, los tipos de redacción de los contenidos escritos, etcétera.

En el proyecto del Museo del Desierto, por ejemplo, podemos encontrar definiciones que nos permiten establecer diferencias y similitudes entre museos comunitarios, museos interactivos y museos al aire libre, así como definiciones referidas a museos de historia natural o museos vivos. En otros casos, el Museo de Palenque o el Museo del Pueblo Maya, vemos el tratamiento, en cuanto a la redacción, contenido y extensión, que se le dio a los textos.

\section{La tercera: recursos y herramientas museográficas}

La clasificación de la información en este apartado nos permite conocer, principalmente, tanto de herramientas como de los recursos museográficos que han sido utilizados para resolver problemas precisos de presentación museográfica. Para esto, el Programa MMM nos ofrece los datos en los que podemos conocer, por ejemplo, cuáles son esas herramientas museográficas y cómo están construidas, en qué situaciones y en qué condiciones han sido utilizadas, qué tan operativas son, y de igual manera saber qué son los recursos 

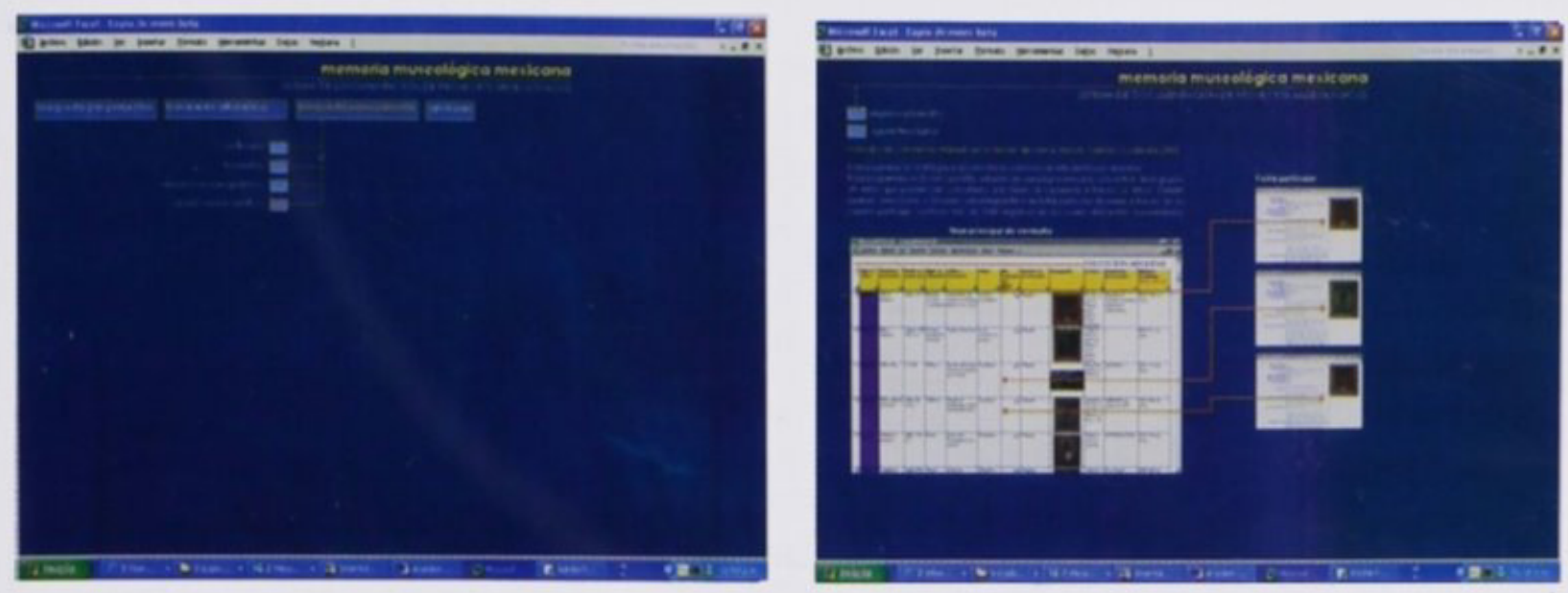

museográficos, cómo han sido empleados y en qué circunstancias, cuáles las ventajas y desventajas en su uso, etcétera.

El programa MMM permite el acceso a esta información por medio de un menú de búsqueda especializada gracias al cual podemos acceder a formatos, modelos o esquemas.

Por ejemplo, las herramientas utilizadas por el Museo del Desierto para estimar los costos de producción y montaje de las exposiciones, las que fueron perfeccionadas en el Proyecto del Museo Arocena, o los formatos de guiones que fueron utilizados hace algunos años en el Museo de Palenque y cómo han evolucionado y se les ha perfeccionado hasta nuestros dias. Para esto, nuestra definición de herramienta hace referencia tanto a los instrumentos que sirven para resolver una necesidad práctica específica (extensión de la mano del hombre) como a las metaherramientas, o sea, aquellas herramientas con las que podemos construir, al mismo tiempo, otras herramientas, característica distintiva y única de los seres humanos y que son, por lo tanto, una especie de extensión de la mente, tomando las palabras de Jorge González, que ayudan a resolver problemas particulares detectados en la realidad. Y, la definición de recurso museográfico, como todo aquel elemento que permite hacer más explicito y entendible los contenidos de las exposiciones, que posibilite la comunicación de los contenidos y agrupaciones discursivas a los públicos visitantes.

\section{La cuarta: La opinión generada}

En esta última se concentra la información que tiene que ver, básicamente, con las variadas opiniones que se han generado, en diversos sectores de la sociedad, en torno a la "construcción" de cada nuevo museo.

Nos permite saber, por ejemplo, qué se dijo en el momento de la creación del museo o qué se ha dicho con el paso del tiempo. Conocer, de igual manera, qué opinan los diversos sectores: profesionistas, especialistas y medios de comunicación respecto de sus exposiciones y de sus colecciones, qué opinan de sus maneras de interpretar y representar museográficamenti is temas y colecciones. 

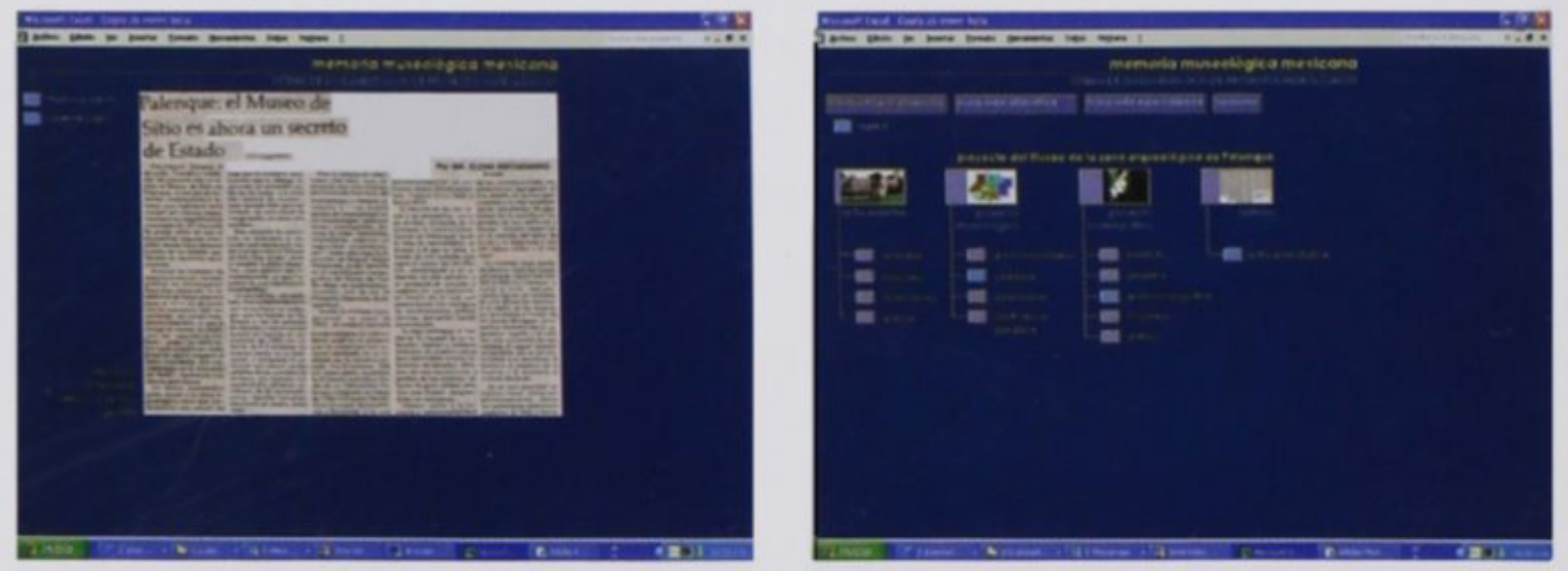

Para ello el programa MMM permite el acceso a la información, principalmente hemerográfica, en la que se contienen diversas opiniones generadas a lo largo del tiempo. Encontramos por ejemplo, en el caso del Museo de Palenque, una nota periodística que, ante el hecho de que nunca se le reinauguró de manera oficial, se pensaba que el museo era una especie de "secreto de Estado". En otros casos, como el Museo del Pueblo Maya, del Desierto, o de Historia Mexicana, la información evidencia que predominan los comportamientos políticos por sobre los culturales ya que en todos ellos su inauguración oficial fue realizada el último día de la gestión de cada gobernante (Salinas y Zedillo, respectivamente) y, en el caso del Museo del Pueblo Maya, el recorrido inaugural no tardó más de unos cuantos minutos y tuvo la misma importancia que la inauguración de un puente peatonal. En otros casos las opiniones apuntan a aspectos más particulares, por ejemplo, reflexionar si por el costo de producción que implicó la creación del Museo del Desierto éste debió realizarse o, si esa cantidad debió invertirse en otro tipo de obra social.

Independientemente del tipo de comentario, el rescate y valoración de esta información tiene como intención permitirnos contar con elementos a partir de los cuales se pueda hacer la reconstrucción más completa posible de los procesos realizados. Estos sistemas permiten rescatar la nota original para evitar la descontextualización de la información, al tiempo que se convierte en otra manera más de obtener la evaluación de los museos y sus exposiciones.

Concluyendo, la intención del Programa MMM es que se convierta en un instrumento que permita conocer las maneras en que abordamos a la museografia y a sus procesos. La sistematización de la información contenida en esta memoria nos permitirá, además, la posibilidad de realizar análisis desde perspectivas interdisciplinares y transdisciplinares mucho más precisas y reales que nos ayuden a reflexionar y proponer teorías y sistemas sobre procesos, métodos y prácticas que puedan ser utilizados en la construcción de la museología mexicana en los próximos años.

El Programa MMM nos permite encontrar conjuntos de información que al ser sistematizados y categorizados pueden convertirse en metodologías (procesos y técnicas 
para llevar a cabo una actividad) que nos ayudarán, entre otros, a resolver problemas propios de la planeación museográfica, como lo son los que corresponden a la creación de exposiciones tanto permanentes y temporales, como a aquellas que nacen a partir de los objetos de colección o de colecciones de ideas, o también a aquellas que tipológicamente corresponden a museos de arte y de historia y, por último, a procesos y políticas que deben desarrollarse cuando, por ejemplo, el origen de los recursos (tanto los financieros como sus colecciones e inmueble) es privado.

Para finalizar y retomando como siempre a Maturana ${ }^{3}$, este sistema conceptual nos permite deducir, además, otros fenómenos no considerados explícitamente en la proposición original, o sea, nos permite generar otros observables que forman parte del mismo proceso y que en el futuro pueden ser abordados para complementar esta información, como son, por ejemplo: las actividades que corresponden a la gestión y operación de los museos, los programas de servicios educativos y de integración para transformar a los visitantes en usuarios, los programas de difusión o de restauración de sus colecciones o, simplemente, las tareas que corresponden al proyecto de arquitectura o de restauración de un inmueble.

Nos permitirá, en suma, enriquecer nuestras maneras de evaluar museos existentes y mejorar enormemente las formas de hacer museos en el futuro.

3 Humberto Maturana y Francisco Varela. El árbol del conocimiento, 1997. Desarrolla Maturana más fondo estos conceptos en sus libros: La realidad: ¿objetiva o construida?, tomo I y II. Barcelona; Ed. Anthropos, UIA, 1997. 
